\title{
Impact of Age on Survival after Partial Portal Vein Arterialization for the Treatment of Post-Hepatectomy Liver Failure in a Rat Model
}

Matteo Novello ${ }^{1}$, Alessandra Zullo ${ }^{1}$, Laura Niccoli ${ }^{1}$, Michele Ruggiero ${ }^{1}$, Raffaele Grande ${ }^{1}$, Marco Cannistrà ${ }^{1}$, Francesco Vito Mandarino ${ }^{1}$, Lorenza Puviani ${ }^{1}$, Giuseppe Cavallari ${ }^{1}$ and Bruno Nardo ${ }^{2 \star}$

${ }^{1}$ Biomedical Research Center, S. Orsola-Malpighi Hospital Bologna, Italy

${ }^{2}$ Department of Specialistic Medicine, Diagnostic and Experimental Sciences, S. Orsola-Malpighi Hospital, University of Bologna, Italy

"Corresponding Author: Bruno Nardo, Department of Specialistic Medicine, Diagnostic and Experimental Sciences, S. Orsola-Malpighi Hospital, University of Bologna, Italy, Tel: +39-3470783648; E-mail: bruno.nardo @unibo.it

Received date: July 24, 2017; Accepted date: September 26, 2017; Published date: September 28, 2017

Copyright: $(2017$ Novello M, et al. This is an open-access article distributed under the terms of the Creative Commons Attribution License, which permits unrestricted use, distribution, and reproduction in any medium, provided the original author and source are credited.

\section{Abstract}

Introduction: Post-operative liver failure (PLF) occurs in approximately $10 \%$ of patients undergoing major hepatectomy. Partial portal vein arterialization (PPVA) enhances the regenerative capacity of the resected liver. The aim of this study was to investigate the impact of age on survival after PPVA for the treatment of post-operative liver failure in a rat model.

Materials and Methods: 24 rats underwent extended liver resection, that leaded to PLF. 12 rats were divided in 2 groups treated with PPVA: group 1a-young rats $(n=6$, age 2 months) and group 2a-old rats ( $n=6$, age 30 months). Two control groups of rats of the same age were not treated with PPVA: group $1 \mathrm{~b}$-young and group $2 \mathrm{~b}$-old.

Results: On postoperative day 7, no significant differences were observed among all groups in terms of ALT levels, prothrombin activity and serum creatinine. As for the liver regeneration markers, the level of mitotic index was greater in the groups treated with PPVA compared to the control groups (without significant differences between young and old groups). The $75 \%(9 / 12)$ of the rats treated with PPVA survived up to 7 days, with no significant differences between young (5/6) and elderly rats $66.7 \%(4 / 6)$.

Conclusion: PPVA treatment had the same beneficial effect both in young and old rats.

Keywords Hepatectomy; Liver failure; Portal vein arterialization

\section{Introduction}

Extended liver resection may result in postoperative liver failure (PLF) that can be fatal [1]. In some cases liver transplantation is suggested but the organ is not always available. PLF occurs in approximately $10 \%$ of patients undergoing major hepatectomy and the main risk factors are the presence of comorbid conditions, pre-existent liver disease and small remnant liver volume [1]. Moreover, it acknowledged that with increasing age, the liver tissue becomes more sensitive to ischemia-reperfusion injury and its regenerative capacity is reduced [2]. There is experimental [3-6] and clinical [7-13] evidence that the liver hyper-oxygenation through the partial portal vein arterialization (PPVA) enhances the regenerative capacity of the resected liver. This event is probably due to the improvement of the microcirculation flow and the tissue oxygen supply. Indeed, this process would satisfy the increased energy demand occurring during the liver regeneration by favouring the oxidative metabolism of hepatocytes $[14,15]$. Thus, this study aimed to assess whether age may be a major determinant of overall survival after PPVA procedure for the treatment of post-operative liver failure (PLF) in a rat model.

\section{Materials and Methods}

A total of 24 male Sprague-Dawley rats weighing 200 to $430 \mathrm{~g}$ underwent extended liver resection $(85 \%)$ which was performed by removing the median, left and caudate lobes. This procedure leaded to postoperative liver failure (PLF) in all the 12 rats which were divided in 2 study groups treated with PPVA: group 1a-young rats $(n=6$, age 2 months, weight 200-300 g) and group 2a-old rats ( $\mathrm{n}=6$, age 30 months, weight 350-430 g). Two control groups of rats of the same age were not treated with PPVA: group 1b-young and group 2b-old. Under enflurane anesthesia, the abdomen of the study group's rats was surgically opened and a left nephrectomy performed. A 24-G polyethylene tube was subsequently placed to connect the left renal artery to the distal portion of the splenic vein but temporarily closed with a nontraumatic clamp. Afterwards, the spleen was removed and a partial hepatectomy performed. Finally, the shunt was opened by removing the clamp and the peritoneal cavity was closed in two layers. At the end of the operation, $1.0 \mathrm{~mL}$ of heparinized physiological saline solution was administered intravenously.

We evaluated the 7-day postoperative survival rate. At the time of death, blood samples $(1 \mathrm{~mL})$ collected from the portal vein were immediately assessed for gas content (OSM3 blood gas analyzer, Radiometer, Copenhagen, Denmark) and blood samples (1 $\mathrm{mL})$ obtained from the inferior vena cava were used to measure serum alanine aminotransferase (ALT), prothrombin time (PT) and serum creatinine (SCr). Formalin-fixed sections were stained with hematoxylin-eosin, whereas ethanol-fixed sections were immunohistochemically stained to reveal mitotic index.

Animal care and experimental procedures were approved by the institutional ethical committee and conducted according to the 
Citation: Novello M, Zullo A, Niccoli L, Ruggiero M, Grande R (2017) Impact of Age on Survival after Partial Portal Vein Arterialization for the Treatment of Post-Hepatectomy Liver Failure in a Rat Model. J Liver 6: 221. doi:10.4172/2167-0889.1000221

Page 2 of 3

guidelines for the care and use of laboratory animals approved by our institution.

Statistical differences between groups were analyzed by two-ways analysis of variance. Survivals were evaluated using Kaplan-Meier curves with differences assessed with the log-rank test. Statistical analysis was performed by running the SPSS statistical package on a personal computer. Data are reported as mean values with standard errors. Two-tailed $\mathrm{P}$ values of less than 0.05 were considered as significant.

\section{Results}

As expected, in young and old rats PPVA treatment induced an increase in $\mathrm{O} 2$ partial pressure $(70 \pm 1.8$ vs. $70 \pm 1.7 \mathrm{mmHg}$ respectively) and oxygen saturation $(89.8 \pm 2.4$ vs. $88 \pm 2.6 \mathrm{mmHg}$, respectively) with a concomitant decrease in $\mathrm{CO} 2$ partial pressure $(37.4 \pm 2.6$ vs. $39.0 \pm 2.5 \mathrm{mmHg}$, respectively). These parameters were registered from the portal blood on postoperative day 7 suggesting that the arterial venous shunt remained patent and functional for such experimental time. Furthermore, no significant differences were observed among all groups in terms of ALT levels, prothrombin activity and serum creatinine up to seven days postoperatively. As for the liver regeneration markers, we detected a greater level of mitotic index in the groups treated with PPVA compared to the control groups (without significant differences between young and old) (Table 1).

\begin{tabular}{|l|l|l|l|l|}
\hline \multirow{2}{*}{ Parameter } & \multicolumn{2}{l|}{ PPVA treated } & \multicolumn{2}{l|}{ PPVA non treated } \\
\cline { 2 - 5 } & Group 1a-young rats & Group 2a- old rats & Group 1b-young rats* & Group 2b-old rats^ \\
\hline Basal: ALT (U/L) & $21+0.4$ & $22+0.7$ & $22+0.3$ & $22+0.5$ \\
\hline At sacrifice: ALT (U/L) & $45 \pm 0.6$ & $48 \pm 0.8$ & 50 & 53 \\
\hline Basal: PT (\%) & $91+3$ & $89+4$ & $90+3$ & $91+2$ \\
\hline At sacrifice: PT (\%) & $90 \pm 4$ & $84 \pm 4$ & 86 & 79 \\
\hline Basal SCr (mg/dL) & $0.87+0.1$ & $0.88+0.2$ & $0.89+01$ & $0.88+03$ \\
\hline At sacrifice: SCr (mg/dL) & $0.90 \pm 0.1$ & $0.93 \pm 0.2$ & 0.90 & 0.95 \\
\hline$N^{\circ}$ mitosis/mm2 & 34.5 & 29.2 & 22.4 & 20.3 \\
\hline Note: ${ }^{* 2}$ survivors; ^1 survivor. & & & \\
\hline
\end{tabular}

Table 1: Biochemical parameters and mitotic index among young and old rats of both study and control groups.

\begin{tabular}{|l|l|l|l|l|}
\hline \multirow{2}{*}{ Weight (gr) } & PPVA treated & \multicolumn{2}{l|}{ PPV non treated } \\
\cline { 2 - 6 } & Group 1a-young rats & Group 2a-old rats & Group 1b-young rats & Group 2b- old rats^ $^{*}$ \\
\hline Rat & $200-300$ & $350-430$ & $200-300$ & $350-430$ \\
\hline Resected liver & 7.8 & 11.2 & 8.1 & 11.5 \\
\hline Remnant liver & 2.2 & 2.8 & 1.9 & 2.5 \\
\hline Preoperative liver & 10 & 14 & 10 & 14 \\
\hline Liver at sacrifice & 6 & 7.8 & 4 & 5.2 \\
\hline Note: *2 survivors; ^1 survivor & & & & \\
\hline
\end{tabular}

Table 2: Liver weights: Differences between control and PPVA treated groups.

After extensive hepatic resection (85\% of the liver), the overall survival at 7 days was only $25 \%(3 / 12)$ in rats not PPVA treated without significant differences regarding age $(33.7 \%, 2 / 6$ young vs. $16.7 \%, 1 / 6$ elderly). In contrast, the $75 \%(9 / 12)$ of the rats treated with PPVA survived up to 7 days $(\mathrm{p}<0.05)$, with no significant differences between young $83.3 \%(5 / 6)$ and elderly rats $66.7 \%(4 / 6)$.

Moreover, the liver weights at sacrifice of the two PA treated groups were significantly greater than that of the two respective control groups (group $1 \mathrm{a}=6 \mathrm{~g}$ vs. group $1 \mathrm{~b}=4 \mathrm{~g}$ and group $2 \mathrm{a}=7.8 \mathrm{~g}$ vs. group $2 \mathrm{~b}=5.2 \mathrm{~g}$; $\mathrm{p}<0.05$ ) (Table 2).

\section{Discussion}

Mortality rates after critical major hepatectomy have been reported to be as high as $30 \%$ with PHLF representing one of the most dreadful complications [16]. The removal of large portions of liver parenchyma is sometimes necessary to fully excise the neoplastic tissue especially for biliary tract tumors. Aside from cancer surgical treatment, extended hepatic resections are also necessary in case of severe injury to the liver parenchyma (e.g., trauma). In 2011, the International Study Group of Liver Surgery (ISGLS) defined PHLF as "a post-operatively acquired deterioration in the ability of the liver to maintain its 
synthetic, excretory, and detoxifying functions, which are characterized by an increased INR and concomitant hyperbilirubinemia on or after postoperative day 5" [17]. However, the severity of its clinical features ranges from mild temporary hepatic insufficiency to fulminant hepatic failure. During the pre-operative assessment in the intent to reduce postoperative morbidity and mortality it is important the evaluation of risk factors like male gender, obesity, diabetes, neoadjuvant treatment with chemotherapy and underlying cirrhosis. The complication severity leading to exitus is related to the quantity of parenchyma removed, but the patient's age plays an important role too. Over the years, the liver parenchyma becomes more sensitive to ischemia-reperfusion injury and its regenerative capacity tends to reduce [14]. The ischemic preconditioning and intermittent clamping by Pringle maneuver are the only intraoperative strategies used during liver resections to reduce the ischemia-reperfusion injury and consequently the risk of postoperative liver failure. Furthermore, the use of PPVA has been widely carried out in clinical practice as a bridge procedure to reduce the risk of acute liver failure (ALF) and to guarantee a better chance for long-term survival. It is well known that the portal blood represents approximately $75 \%$ of the total blood flow to the liver, has an oxygen partial pressure $<40 \mathrm{mmHg}$ and a low hydrostatic pressure (5-10 $\mathrm{mmHg}$ ).

Since the PPVA raises the hydrostatic pressure of the portal blood and especially the percentage of oxygen saturation to values similar to those found in the arterial blood, we assume that this strong oxygen increase satisfies the high metabolic demand of the regenerating hepatocytes by favouring the oxidative metabolism. The exact mechanism by which PPVA stimulates liver regeneration is not currently known and further studies should be planned. However, it appears reasonable to hypothesize that the extra oxygen supplied to the liver through the arterovenous shunt [15] mediates the regeneration promotion.

\section{Conclusion}

This study revisited the benefits of PPVA in the promotion of liver regeneration demonstrating that such surgical procedure was protective against PLF induced by hepatectomy in a population of young and old rats. The presence of an additional supply of oxygenated blood in the portal venous system following the PPVA procedure has had positive effects on energy metabolism and has led to a significantly higher survival of arterialized rats. The PPVA treatment had the same beneficial effects on both young and old rats, with no statistically significant differences. Our conclusion suggests that even the elderly liver responds well to the regenerative stimulation induced by the PPVA shunting. However, further large-scale studies and applications are needed to confirm the effectiveness of this procedure.

\section{References}

1. Balzan S, Belghiti J, Farges O, Ogata S, Sauvanet A, et al. (2005) The "50-50 Criteria" on oostoperative day 5 an accurate predictor of liver failure and death after hepatectomy. Ann Surg 6: 824-829.
2. Cannistrà M, Grande R, Ruggiero M, Novello M, Zullo A, et al. (2016) Resection of hepatocellular carcinoma in elderly patients and the role of energy balance). Int J Surg. 33: S119-S125.

3. Shimizu Y, Miyazaki M, Shimizu H, Ito H, Nakagawa K, et al. (2000) Beneficial effects of arterialization of the portal vein on extended hepatectomy. Br J Surg 87: 784-789.

4. Fan YD, Praet M, Van Huysse J, Lelie B, De Hemptinne B, et al. (2012) Effects of portal vein arterialization on liver regeneration after partial hepatectomy in the rat. Liver Transpl 8:146-152.

5. Nardo B, Puviani L, Caraceni P, Pacilè V, Bertelli R, et al. (2016) Portal vein arterialization for the treatment for post-resection acute liver failure in the rat. Transplant Proc 38: 1185-1191.

6. Nardo B, Montalti R, Puviani L, Pacilè V, Beltempo P, et al. (2006) An experimental pilot study on controlled portal vein arterialization with an extracorporeal device in the swine model of partial liver resection and ischemia. Int J Artif Organs 29: 912-918.

7. Ozeki Y, Umemoto T, Tateyama K, Katagiri Y, Katayama M, et al. (1997) Partial portal arterialization for dearterialized liver after hepatectomy. $\mathrm{Br}$ J Surg 84:1011.

8. Tanabe G, Kawaida K, Hamanoue M, Kihara K, Hirata S, et al. (1999) Treatment for accidental occlusion of the hepatic artery after hepatic resection: report of two cases. Surg Today 29: 268-275.

9. Nardo B, Caraceni P, Montalti R, Puviani L, Bertelli R, et al. (2005) Portal vein arterialization: a new surgical option against acute liver failure? Transplant Proc. 37: 2544-2549.

10. Tsivian M, Neri F, Prezzi D, Puviani L, Pacile V, et al. (2007) Portal vein arterialization in hepatobiliary surgery and liver transplantation. Transplant Proc 39: 1877-1878.

11. Nardo B, Vaccarisi S, Pellegrino V, Cannistrà M, Barcellona E, et al. (2011) Extracorporeal portal vein arterialization in man after extended hepatectomy to prevent acute liver failure: a case report. Transplant Proc 43: 1193-1195.

12. Bhangui P, Salloum C, Lim C, Andreani P, Ariche A, et al. (2014) Portal vein arterialization: a salvage procedure for a totally de-arterialized liver. The Paul Brousse Hospital experience. HPB (Oxford) 16: 723-738.

13. Qiu J, Chen S, Pankaj P, Wu H. (2014) Portal vein arterialization as a bridge procedure against acute liver failure after extended hepatectomy for hilar cholangiocarcinoma. Surg Innov 21: 372-375.

14. Cavallari G, Bonaiuto E, Tsivian M, Vaccarisi S, Nardo B, et al. (2014) Partial portal vein arterialization in acute liver failure. Updates Surg. 66:183-187.

15. Nardo B, Caraceni P, Puviani L, Pertosa AM, Domenicali M, et al. (2006) Successful treatment of CCl4-induced acute liver failure with portal vein arterialization in the rat. J Surgical Res 135: 394-401.

16. Kauffmann R, Fong Y. (2014) Post-hepatectomy liver failure. Hepatobiliary Surg Nutr 3: 238-246.

17. Rahbari NN, Garden OJ, Padbury, Brooke-Smith M, Crawford M, et al (2011) Posthepatectomy liver failure: a definition and grading by the International Study Group of Liver Surgery (ISGLS). Surgery 149: 713-24. 